\title{
The role of the intra-abdominal view in complicated intra-abdominal infections
}

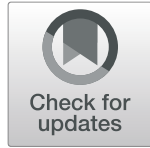

\author{
Matti Tolonen ${ }^{1 *} \mathbb{D}$, Ville Sallinen ${ }^{1,2}$, Ari Leppäniemi ${ }^{1}$, Minna Bäcklund ${ }^{3}$ and Panu Mentula ${ }^{1}$
}

\begin{abstract}
Background: The prognostic role of what a surgeon observes in the abdomen of patients with complicated intraabdominal infection (CIAl) is largely unknown. The aim of this prospective study was to systemically analyze components of the intra-abdominal view (IAV) and their association to severe complicated intra-abdominal sepsis (SCIAS) or mortality.

Methods: The study cohort consisted of adult patients with cIAI. The operating surgeon filled a paper form describing the intra-abdominal view. Demographics, operative details, and preoperative physiological status were collected. Descriptive, univariate, and multivariate statistical analyses were performed, and a new score was developed based on regression coefficients. The primary outcome was a composite outcome of SCIAS or 30-day mortality, in which SCIAS was defined as organ dysfunctions requiring intensive care unit admission.
\end{abstract}

Results: A total of 283 patients were analyzed. The primary outcome was encountered in 71 (25\%) patients. In the $I A V$, independent risk factors for the primary outcome were fecal or bile as exudate (odds ratio (OR) 1.98, 95\% confidence interval 1.05-3.73), diffuse peritonitis (OR 2.15, 1.02-4.55), diffuse substantial redness of the peritoneum (OR 5.73, 2.12-15.44), and a non-appendiceal source of CIAI (OR 11.20, 4.11-30.54). Based on these factors, an IAV score was developed and its performance analyzed. The area under the receiver operating characteristic for the IAV score was 0.81 . The IAV score also correlated significantly with several outcomes and organ dysfunctions.

Conclusions: The extent of peritonitis, diffuse substantial redness of the peritoneum, type of exudate, and source of infection associate independently with SCIAS or mortality. A high IAV score associates with mortality and organ dysfunctions, yet it needs further external validation. Combining components of IAV into comprehensive scoring systems for cIAl patients may provide additional value compared to the current scoring systems.

Trial registration: The study protocol was retrospectively registered on April 4, 2016, right after the first enrolled patient at Clinicaltrials.gov database (NCT02726932).

Keywords: Intra-abdominal infection, Secondary peritonitis, Emergency surgery, Sepsis, Intra-abdominal view, Severe peritonitis

\section{Background}

Perforation in the gastrointestinal tract results in complicated intra-abdominal infection (cIAI) that may present either as localized peritonitis with or without abscess formation or as diffuse peritonitis [1]. The intra-abdominal view (IAV) in cIAI is traditionally classified into generalized or local and clear, purulent, or fecal according to the appearance of the exudate in the

\footnotetext{
* Correspondence: matti.tolonen@hus.fi

${ }^{1}$ Abdominal Center, Department of Abdominal Surgery, Helsinki University Hospital and University of Helsinki, Haartmaninkatu 4, 00290 Helsinki, Finland Full list of author information is available at the end of the article
}

abdomen [2]. Other classifications separate communityor hospital-acquired cIAIs and postoperative or non-postoperative IAIs [3].

In a cIAI, various other findings are often seen in the abdomen, for example, bowel dilatation, bile as exudate, various degrees of fibrin coverage, and redness in the parietal and/or visceral peritoneum.

Even though all surgeons know that a large variety in the IAV exists in patients with cIAI, IAV has not received much attention in previous literature or clinical work. As an example, a patient with acute perforated diverticulitis and a small amount of pus in the pelvis and

(C) The Author(s). 2019 Open Access This article is distributed under the terms of the Creative Commons Attribution 4.0 International License (http://creativecommons.org/licenses/by/4.0/), which permits unrestricted use, distribution, and reproduction in any medium, provided you give appropriate credit to the original author(s) and the source, provide a link to the Creative Commons license, and indicate if changes were made. The Creative Commons Public Domain Dedication waiver (http://creativecommons.org/publicdomain/zero/1.0/) applies to the data made available in this article, unless otherwise stated. 
among small bowel loops, in an otherwise perfectly normally looking abdomen, would be classified as Hinchey III [4]. The same grade of classification would be used in an abdomen full of pus, with dilated bowel, extensive fibrin coverage, and substantially red peritoneal surfaces all over the abdomen. It seems intuitive that recovery paths of these patients would be different and, therefore, all Hinchey III patients cannot be considered equal only based on the existence of purulent peritonitis $[5,6]$.

There is a wide range of disease severity in patients with cIAI. A term severe complicated intra-abdominal sepsis (SCIAS) to describe sepsis-related organ dysfunctions combined with cIAI specifically due to a disruption in the gastrointestinal tract was introduced in an article by Kirkpatrick et al. [7]. A variety of risk factors for SCIAS or mortality have been identified, preoperative sepsis with associated organ dysfunctions and septic shock being the most important ones [3, 8-10]. Other crucial factors include the physiological reserve of the patient, comorbidities, immunosuppressive medications, and the anatomical derangement caused by the acute disease. There are multiple scoring systems, general and cIAI-specific combining these factors to predict severe outcomes but no one system works satisfyingly [11]. These systems include limited data of the IAV.

The aim of this prospective study was to systematically analyze components of IAV and their association to SCIAS or 30-day mortality.

\section{Methods}

\section{Patients and setting}

This study was conducted as a prospective cohort study in a single academic center that serves both as a secondary and a tertiary referral center. Institutional ethics committee and review board approved the study design. A written informed consent was obtained from all the patients included in the study. The study period was 2 years, starting on 31 March 2016 and ending on 31 March 2018.

Eligible patients were adult (over 18 years old) patients undergoing surgical intervention because of a cIAI. Patients with pancreatitis, acute mesenteric ischemia, or trauma were excluded. During the study period, electronic operating room logs were manually browsed for all abdominal emergency operations to verify that all patients with a cIAI were identified.

\section{Intra-abdominal view}

Data regarding intra-abdominal findings was collected on a paper sheet form filled by the operating surgeon after the operation (Additional file 1). In the form, the abdomen was divided into six areas: right and left upper supramesocolic area, right and left lower and paracolic area, mid-abdomen small bowel area, and pelvic area (below the promontorium). Small and/or large bowel dilatation was recorded as well as the type of exudate (clear, purulent, fecal, bile) by area. If any area contained fecal or bile exudate, the peritonitis was classified as fecal or bile peritonitis, respectively. If both were present, the peritonitis was classified according to the predominant exudate. In addition, the amount of fibrin by area (none, mild, or substantial) and redness of the peritoneum by area (none, mild, or substantial) were recorded. Further, the localization of infection in the peritoneum by area (parietal and/or visceral, excluding the pelvic area) was recorded. The difference between mild and substantial finding was a subjective estimation based on clinical judgment and experience.

\section{Definitions}

Comorbidities were classified according to the Charlson Comorbidity Index [12]. The classification of sepsis was recorded as in the Sepsis-III guidelines, i.e., sepsis is an acute change of total Sequential Organ Failure Assessment (SOFA) score two or more and septic shock is sepsis with persisting hypotension requiring vasopressors to maintain mean arterial pressure $\geq 65 \mathrm{mmHg}$ and a persistent serum lactate level $>2 \mathrm{mmol} / \mathrm{l}$ despite adequate volume resuscitation [10]. Other reported scores were the Mannheim Peritonitis Index, World Society of Emergency Surgery (WSES) Sepsis Severity Score for patients with cIAIs, Acute Physiology And Chronic Health Evaluation II (APACHE-II), Clavien-Dindo complication classification, and the SOFA scores in patients admitted to the ICU $[2,13-16]$. For the Clavien-Dindo classification, the most serious in-hospital complication was used. Preoperative organ dysfunctions (grade IV) or antibiotic treatment for cIAI were not documented as complications. Only new onset organ dysfunctions or complications that significantly contributed to the worsening of preoperative organ dysfunctions were classified as grade IV [17]. Immunosuppression was defined according to the WSES Sepsis Severity Score, i.e., chronic use of glucocorticoids, immunosuppressive medication, chemotherapy within 30 days, or lymphatic disease. The primary outcome was a composite outcome of SCIAS or 30-day mortality. SCIAS translated to ICU admission due to organ dysfunctions. Similar definition has also been used in a recent study [11].

\section{Statistical analyses}

Descriptive statistics are demonstrated in number, percentage, mean, median, and interquartile range (IQR), where appropriate. Univariate analyses were made using binary logistic regression for categorical variables, Mantel-Haenszel linear-by-linear association chi-squared test for ordinal variables, and Mann-Whitney $U$ test or Kruskal-Wallis test for continuous variables without normal distribution, where appropriate. Nonparametric 
correlations between continuous variables were tested with Spearman's rho test. Multivariate analysis was conducted using binary logistic regression. Multivariate goodness-of-fit was tested using Hosmer-Lemeshow test, and model performance was tested using Nagelkerke $R^{2}$. Variables for multivariate analysis were chosen from the results of the univariate analysis. After logical reclassification in some variables, the most significant variable from each IAV component (perforated organ, extent of peritonitis, bowel dilatation, type of exudate, fibrin coverage, and redness in peritoneum) was chosen. Different logistic regression models were tested with different combinations of variables, and the model with the highest Nagelkerke $R^{2}$ value was chosen. The scoring system was built according to the regression coefficients in the logistic regression equation. A receiver operating characteristic (ROC) curve was plotted, and area under ROC curve (AUROC) calculated. Two-tailed $P$ value below 0.05 was considered significant, and odds ratio (OR) values are presented with $95 \%$ confidence intervals (CI). Analyses mentioned above were per-

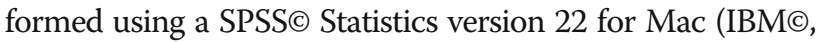
Armonk, NY, USA). Internal validation on logistic model was done with bootstrapping and by calculating optimism-adjusted AUC [18]. This analysis was done using R (R Foundation for Statistical Computing, Vienna, Austria.
URL https://www.R-project.org/.) with pROC package [19] and R function for optimism-adjusted AUC [20].

\section{Power calculation}

Power calculations for this study were based on the primary outcome measure, i.e., SCIAS or 30-day mortality. In order to perform a statistically appropriate multivariate analysis with seven variables, we calculated the need for a minimum of 49 patients with the primary outcome event. Based on previous reports, $10-25 \%$ of patients with cIAI will meet the primary outcome [8, 13]. For power calculation purposes, we estimated that $17 \%$ of cIAI patients would meet the primary outcome event resulting in a need to recruit at least 288 patients.

\section{Results}

During the 2-year study period, there were 657 operated patients with cIAIs filling the inclusion; please see the modified flow diagram (Fig. 1). The most common reasons for not recruiting patients were failure to attempt recruiting and not suspecting cIAI preoperatively.

A total of 283 patients with properly filled IAV form were included and analyzed. Patient characteristics, together with the intra- and postoperative data, are presented in Table 1, describing the study cohort. Briefly,

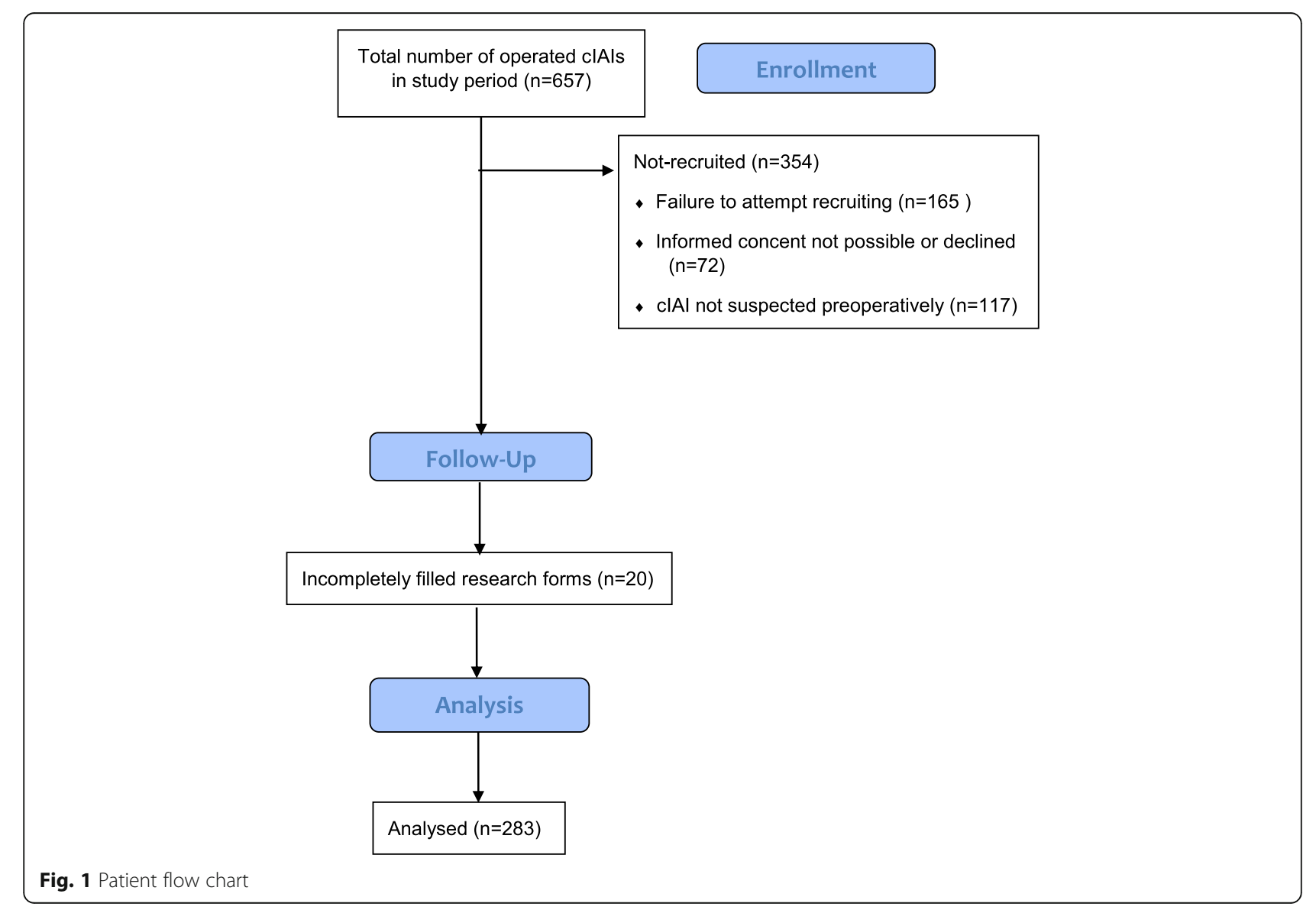


Table 1 Patient characteristics

\begin{tabular}{|c|c|}
\hline Preoperative & $n(\%)($ total $n=283)$ \\
\hline Sex, male & $132(47)$ \\
\hline Age, years & $64(49-74)^{a}$ \\
\hline BMI & $21(19-24)^{a}$ \\
\hline Charlson Comorbidity Index & $1(0-5)^{a}$ \\
\hline Immunosuppression & $54(19)$ \\
\hline \multicolumn{2}{|l|}{ Malignant diseases } \\
\hline Local solid malignant tumor & $35(12)$ \\
\hline Solid metastatic tumor or lymphoma & $45(16)$ \\
\hline \multicolumn{2}{|l|}{ Sepsis classification } \\
\hline No sepsis & $204(72)$ \\
\hline Sepsis & $60(21)$ \\
\hline Septic shock & $19(7)$ \\
\hline Hospital-acquired c|Al & $62(22)$ \\
\hline Intraoperative & $n(\%)$ \\
\hline Delay from symptoms to operation $<24 \mathrm{~h}$ & $79(28)$ \\
\hline \multicolumn{2}{|l|}{ Perforated organ } \\
\hline Gastroduodenal & $32(11)$ \\
\hline Small bowel & $30(11)$ \\
\hline Colorectal & $101(36)$ \\
\hline Appendix & 109 (39) \\
\hline Gallbladder & $11(4)$ \\
\hline \multicolumn{2}{|l|}{ Operation } \\
\hline Laparotomy & $152(54)$ \\
\hline Laparoscopy & $110(39)$ \\
\hline Converted from laparoscopy to laparotomy & $21(7)$ \\
\hline Open abdomen & $5(2)$ \\
\hline Postoperative peritonitis & $38(13)$ \\
\hline Mannheim Peritonitis Index & $26(22-32)^{a}$ \\
\hline WSES Sepsis Severity Score & $6(3-9)^{a}$ \\
\hline APACHE-\| & $9(6-15)^{a}$ \\
\hline Postoperative & $n(\%)$ \\
\hline CRP, highest value, mg/l & $304(244-364)^{\mathrm{a}}$ \\
\hline Reoperations & $36(13)$ \\
\hline Postoperative abscess & $28(10)$ \\
\hline \multicolumn{2}{|l|}{ Clavien-Dindo classification, in-hospital } \\
\hline 0 & $111(39)$ \\
\hline 1 & $47(17)$ \\
\hline 2 & $38(13)$ \\
\hline 3a & $28(10)$ \\
\hline $3 b$ & $25(9)$ \\
\hline $4 a$ & $7(2)$ \\
\hline $4 b$ & $3(1)$ \\
\hline 5 & $24(8)$ \\
\hline Length of hospital stay, days & $6(3-11)^{a}$ \\
\hline
\end{tabular}

Table 1 Patient characteristics (Continued)

\begin{tabular}{ll}
\hline ICU admission & $57(20)$ \\
Renal replacement therapy & $2(1)$ \\
Peak SOFA score at ICU & $8(6-10)^{\mathrm{a}}$ \\
Prolonged (> 12 h) recovery room stay & $25(9)$ \\
Mortality, 30 days & $29(10)$ \\
SCIAS or 30-day mortality & $71(25)$ \\
Mortality, 90 days & $37(13)$ \\
\hline
\end{tabular}

Abbreviations: $B M I$ body mass index, CIAI complicated intra-abdominal infection, WSES World Society of Emergency Surgery, APACHE Acute

Physiology and Chronic Health Evaluation, CRP C-reactive protein, ICU intensive care unit, SCIAS severe complicated intra-abdominal sepsis

${ }^{a}$ Continuous variables are presented as median (interquartile range)

$132(47 \%)$ were male with a median age of 64 years. Sepsis was present in $60(21 \%)$ and septic shock in 19 (7\%) patients preoperatively. The most common sources of cIAI were the appendix $(n=109,39 \%)$ and colorectal $(n$ $=101,36 \%)$. The median length of hospital stay was 6 days, and 57 (20\%) patients were admitted to the ICU with a median peak SOFA score of 8 . All patients admitted to the ICU had a SOFA score of at least 1 . Twenty-nine (10\%) patients died within 30 days, and SCIAS or mortality was encountered in 71 (25\%) patients.

Several different components of the IAV were associated with the primary outcome (Table 2). Some components of the IAV were reclassified according to the results to build the multivariate analysis (marked with “*” in Table 2). Non-appendiceal source of cIAI, diffuse peritonitis (four or more out of six areas), dilatation of the colon only, fecal or bile as exudate, substantial fibrin deposits found in five or more areas, and substantial redness found in four or more areas were chosen for the multivariate analysis. Correlations between continuous variables were tested, and all results were significant $(P$ $<0.001)$. Correlation coefficients for the amount of substantial redness by area were 0.433 for the amount of substantial fibrin by area and 0.385 for the extent of peritonitis by area. For the amount of substantial fibrin by area and extent of peritonitis by area, the correlation coefficient was 0.293 .

In the multivariate binary logistic regression analysis, four components of the IAV were found independently significantly associated with the primary outcome (Table 3). In an ascending OR order, these factors were fecal or bile as exudate, diffuse peritonitis, substantial redness in four or more areas, and a non-appendiceal source. AUROC for this logistic model was 0.812 (95\% CI 0.761$0.865)$. Internal validity of the logistic model was tested with bootstrapping. Distribution of the AUROC value in the bootstrap sample is shown in Additional file 2 . Optimism-adjusted AUROC for the logistic model was 0.802. Hosmer-Lemeshow goodness-of-fit test significance 
Table 2 Univariate binary logistic regression of components of the intra-abdominal view for severe complicated intra-abdominal sepsis (SCIAS) or 30-day mortality

\begin{tabular}{|c|c|c|c|c|c|}
\hline Risk factor & $n(\%)$ & SCIAS or 30-day mortality, $n$ (\%) & OR $(95 \% \mathrm{Cl})$ & $P$ value & $B$ \\
\hline \multicolumn{6}{|l|}{ Perforated organ } \\
\hline Appendix & $109(39)$ & $5(5)$ & Reference & $<0.001$ & \\
\hline Gallbladder & $11(4)$ & $4(36)$ & $11.89(2.60-54.42)$ & 0.001 & 2.475 \\
\hline Colorectal & $101(36)$ & $37(37)$ & $12.03(4.49-32.18)$ & $<0.001$ & 2.487 \\
\hline Gastroduodenal & $32(11)$ & $12(38)$ & $12.48(3.96-39.33)$ & $<0.001$ & 2.524 \\
\hline Small bowel & $30(11)$ & $13(43)$ & $15.91(5.03-50.33)$ & $<0.001$ & 2.767 \\
\hline Non-appendiceal source* & $174(62)$ & $66(38)$ & $12.71(4.93-32.81)$ & $<0.001$ & 2.542 \\
\hline \multicolumn{6}{|c|}{ Extent of peritonitis by number of affected areas } \\
\hline 1 & $30(11)$ & $4(13)$ & Reference & 0.001 & \\
\hline 2 & $47(17)$ & $7(15)$ & $1.14(0.30-4.28)$ & 0.849 & 0.129 \\
\hline 3 & $29(10)$ & $1(3)$ & $0.23(0.02-2.21)$ & 0.204 & -1.460 \\
\hline 4 & $32(11)$ & $8(25)$ & $2.17(.58-8.13)$ & 0.252 & 0.773 \\
\hline 5 & $32(11)$ & $7(22)$ & $1.82(0.47-6.99)$ & 0.383 & 0.599 \\
\hline 6 & $113(40)$ & $44(39)$ & $4.15(1.35-12.69)$ & 0.013 & 1.422 \\
\hline Diffuse peritonitis ( $\geq 4$ areas)* & $177(63)$ & $59(33)$ & $3.92(1.99-7.71)$ & $<0.001$ & 1.365 \\
\hline \multicolumn{6}{|l|}{ Bowel dilatation } \\
\hline No bowel dilatation & $164(58)$ & $35(21)$ & Reference & 0.097 & \\
\hline Small bowel only & $77(27)$ & $19(25)$ & $1.21(0.64-2.29)$ & 0.563 & 0.188 \\
\hline Colon only* & $19(7)$ & $8(42)$ & $2.68(1.00-7.17)$ & 0.050 & 0.986 \\
\hline Small bowel and colon & $23(8)$ & $9(39)$ & $2.37(0.95-5.93)$ & 0.065 & 0.863 \\
\hline \multicolumn{6}{|l|}{ Type of exudate } \\
\hline Clear & $16(6)$ & $3(19)$ & Reference & 0.001 & \\
\hline Purulent & $160(57)$ & $27(17)$ & $0.88(0.24-3.30)$ & 0.849 & -0.128 \\
\hline Fecal & $85(30)$ & $30(35)$ & $2.36(0.62-8.95)$ & 0.206 & 0.860 \\
\hline Bile & $22(8)$ & $11(52)$ & $4.33(0.96-19.58)$ & 0.057 & 1.466 \\
\hline Fecal or bile* & $107(38)$ & $41(38)$ & $3.02(1.74-5.26)$ & $<0.001$ & 1.106 \\
\hline \multicolumn{6}{|l|}{ Extent of fibrin by number of affected areas } \\
\hline 0 & $37(13)$ & $15(41)$ & Reference & $<0.001$ & \\
\hline 1 & $77(27)$ & $12(16)$ & $0.27(0.11-0.67)$ & 0.004 & -1.306 \\
\hline 2 & $46(16)$ & $7(15)$ & $0.26(0.09-0.74)$ & 0.120 & -1.335 \\
\hline 3 & $28(10)$ & $3(11)$ & $0.18(0.05-0.69)$ & 0.130 & -1.737 \\
\hline 4 & $31(11)$ & $6(19)$ & $0.35(0.12-1.06)$ & 0.640 & -1.044 \\
\hline 5 & $24(9)$ & $10(42)$ & $1.05(0.37-2.98)$ & 0.930 & 0.047 \\
\hline 6 & $39(14)$ & $18(46)$ & $1.26(0.51-3.12)$ & 0.622 & 0.229 \\
\hline \multicolumn{6}{|c|}{ Amount of substantial fibrin by number of affected areas } \\
\hline 0 & $140(49)$ & $33(24)$ & Reference & 0.016 & \\
\hline 1 & $72(25)$ & $12(17)$ & $0.65(0.31-1.35)$ & 0.246 & -0.433 \\
\hline 2 & $25(9)$ & $6(24)$ & $1.02(0.38-2.78)$ & 0.963 & 0.024 \\
\hline 3 & $19(7)$ & $7(37)$ & $1.89(0.69-5.20)$ & 0.216 & 0.637 \\
\hline 4 & $10(4)$ & $2(20)$ & $0.81(0.16-4.01)$ & 0.797 & -0.210 \\
\hline 5 & $6(2)$ & $4(67)$ & $6.49(1.14-37.01)$ & 0.035 & 1.869 \\
\hline 6 & $11(4)$ & $7(64)$ & $5.67(1.56-20.59)$ & 0.008 & 1.736 \\
\hline Substantial fibrin $\geq 5$ or more areas* & $17(6)$ & $11(65)$ & $6.29(2.2-17.73)$ & $<0.001$ & 1.840 \\
\hline
\end{tabular}


Table 2 Univariate binary logistic regression of components of the intra-abdominal view for severe complicated intra-abdominal sepsis (SCIAS) or 30-day mortality (Continued)

\begin{tabular}{|c|c|c|c|c|c|}
\hline Risk factor & $n(\%)$ & SCIAS or 30-day mortality, $n(\%)$ & OR $(95 \% \mathrm{Cl})$ & $P$ value & B \\
\hline \multicolumn{6}{|l|}{ Extent of redness by number of affected areas } \\
\hline 0 & $17(6)$ & $8(47)$ & Reference & $<0.001$ & \\
\hline 1 & $41(14)$ & $6(15)$ & $0.19(0.05-0.70)$ & 0.012 & -1.646 \\
\hline 2 & $55(19)$ & $7(13)$ & $0.16(0.05-0.57)$ & 0.004 & -1.808 \\
\hline 3 & $32(11)$ & $3(9)$ & $0.12(0.03-0.53)$ & 0.006 & -2.151 \\
\hline 4 & $32(11)$ & $7(22)$ & $0.32(0.09-1.12)$ & 0.074 & -1.155 \\
\hline 5 & $27(10)$ & $7(26)$ & $0.39(0.11-1.42)$ & 0.155 & -0.932 \\
\hline 6 & $78(28)$ & $33(42)$ & $0.83(0.29-2.37)$ & 0.720 & -1.192 \\
\hline \multicolumn{6}{|c|}{ Amount of substantial redness by number of affected areas } \\
\hline 0 & $125(44)$ & $26(21)$ & Reference & $<0.001$ & \\
\hline 1 & $73(26)$ & $10(14)$ & $0.60(0.27-1.34)$ & 0.214 & -0.504 \\
\hline 2 & $34(12)$ & $10(29)$ & $1.59(0.68-3.73)$ & 0.290 & 0.462 \\
\hline 3 & $21(7)$ & $5(24)$ & $1.19(0.40-3.55)$ & 0.755 & 0.174 \\
\hline 4 & $9(3)$ & $5(56)$ & $4.76(1.19-18.99)$ & 0.027 & 1.560 \\
\hline 5 & $3(1)$ & $2(67)$ & $7.62(0.66-87.29)$ & 0.103 & 2.030 \\
\hline 6 & $18(6)$ & $13(72)$ & $9.90(3.24-30.29)$ & $<0.001$ & 2.293 \\
\hline Substantial redness $\geq 4$ or more areas* & $30(11)$ & $20(67)$ & $7.92(3.49-17.97)$ & $<0.001$ & 2.070 \\
\hline Localization of cIAI non-parietal (only visceral) & $49(17)$ & $11(22)$ & $0.84(0.40-1.75)$ & 0.639 & -1.094 \\
\hline
\end{tabular}

Method: enter, all variables categorical. Italics indicate statistical significance

Abbreviations: $O R$ odds ratio, $B$ regression coefficient, $C I A l$ complicated intra-abdominal infection

*Included in multivariate analysis

was 0.36 showing the model had adequate fit. Model performance was tested using Nagelkerke $R^{2}$ with a result of 0.36 .

The IAV score is also presented in Table 3. Regression coefficients were multiplied by 1.3 , and the nearest integer was the score for each variable. AUROC for IAV score predicting SCIAS or 30-day mortality was 0.81 , and it remained unchanged from the original logistic model. As shown in Fig. 2, the IAV score performed nearly as good as other scores in predicting SCIAS or mortality. With the help of the ROC curve (Fig. 2), two cutoff points were chosen to divide patients to low ( $0-2$ points), medium (3-5 points), and high score (6-7 points) groups. These groups were compared for various different outcomes (Table 4).

Because of the dynamic nature of organ dysfunctions, an additional analysis was made to evaluate whether the IAV score could predict the course of organ dysfunction (Fig. 3). Patients with preoperative organ dysfunctions had a higher median IAV score (4, IQR 4-5) compared to patients without organ dysfunctions (3, IQR $1-4), P<0.001$. Of the 204 patients without preoperative organ dysfunctions, $13(6 \%)$ were admitted to the ICU or died, and these patients had a significantly higher IAV score (4, IQR 3-5) compared to the patients who recovered without ICU treatment (3, IQR 1-4), $P=0.008$. On the other hand, patients whose preoperative organ dysfunctions resolved quickly without ICU treatment had a lower IAV score (3, IQR 1-4) compared to the ones who were admitted to the ICU or died (5, IQR 4-6), $P<0.001$.

\section{Subgroup analysis}

Due to the much better prognosis in acute appendicitis as the source of cIAI, we performed a subgroup analysis with a non-appendiceal source of cIAI. There were 174 patients, of which 27 (16\%) died within 30 days, 53 (30\%) were admitted to the ICU, and $66(38 \%)$ had a SCIAS. A multivariate analysis was performed with the Table 3 variables, without source of infection, using the enter method. The results were substantial redness in $\geq 4$ areas (OR 5.09, 95\% CI 1.71-15.13, $P 0.003, B 1.628$ ), fecal or bile as exudate (OR 2.06, 95\% CI 1.06-4.03, $P$ 0.034, $B$ 0.725), and diffuse peritonitis (OR 2.14, 95\% CI 0.97-4.72, P 0.059, B 0.761). Hosmer-Lemeshow test showed a non-significant value of 0.91 and Nagelkerke $R^{2} 0.19$.

\section{Discussion}

This pioneer prospective study of the IAV in patients with cIAI recognized several independent factors associated with SCIAS or 30-day mortality. Based on these factors, an IAV score was developed and its performance further analyzed.

We chose a composite outcome of either ICU admission due to acute organ dysfunctions (=SCIAS) or 30-day mortality as the primary outcome. All patients 
Table 3 Multivariate binary logistic regression of the intra-abdominal view for severe complicated intra-abdominal sepsis (SCIAS) or 30-day mortality

\begin{tabular}{lllll}
\hline Risk factor & SCIAS or 30-day mortality OR $(95 \% \mathrm{Cl})$ & $P$ value & $B$ & IAV score \\
\hline Exudate fecal or bile & $1.98(1.05-3.73)$ & 0.034 & 0.685 & 1 \\
Diffuse peritonitis $(\geq 4$ areas) & $2.15(1.02-4.55)$ & 0.045 & 0.767 & 1 \\
Substantial redness $(\geq 4$ areas) & $5.73(2.12-15.44)$ & 0.001 & 1.745 & 2 \\
Non-appendiceal source & $11.20(4.11-30.54)$ & $<0.001$ & 2.416 & 3 \\
\hline
\end{tabular}

Method: forward LR, all variables categorical

Abbreviations: $O R$ odds ratio, $C I$ confidence interval, $B$ regression coefficient, IAV intra-abdominal view (score $=1.3 \times B$ to nearest integer)

admitted to the ICU had acute organ dysfunctions. With this outcome, all patients with a severe outcome were recognized, since some patients may die without being admitted to the ICU. This may be due to ICU admission refusals, or a more surprising death without diagnosed previous organ dysfunctions.

The results from the multivariate analysis are somewhat expected. A completely new finding was that having substantial redness in four or more out of six areas in the abdomen was independently associated with the primary outcome. Intuitively, it seems logical. The inflammatory response of the peritoneum is characterized by enhanced vascular perfusion, accumulation of macrophages with subsequent attraction of more immune cells, and release of pro- and anti-inflammatory mediators [2123]. The stronger the inflammation process, the more visible the redness of the peritoneum. Patients with appendicitis have substantially better prognosis compared to other cIAIs $[3,13]$. Even when adjusting for other IAV factors, patients having other sources than appendicitis was the risk factor with the highest OR. In future cIAI studies, it should be considered if appendicitis should be studied as a separate entirety with different outcome measures due to a much less severe course of the disease. Having a diffuse, rather than localized cIAI, the risk for SCIAS or 30-day mortality increased threefold. This risk seems obvious and has also been identified by the previously existing scoring systems $[2,13]$. When the type of exudate was

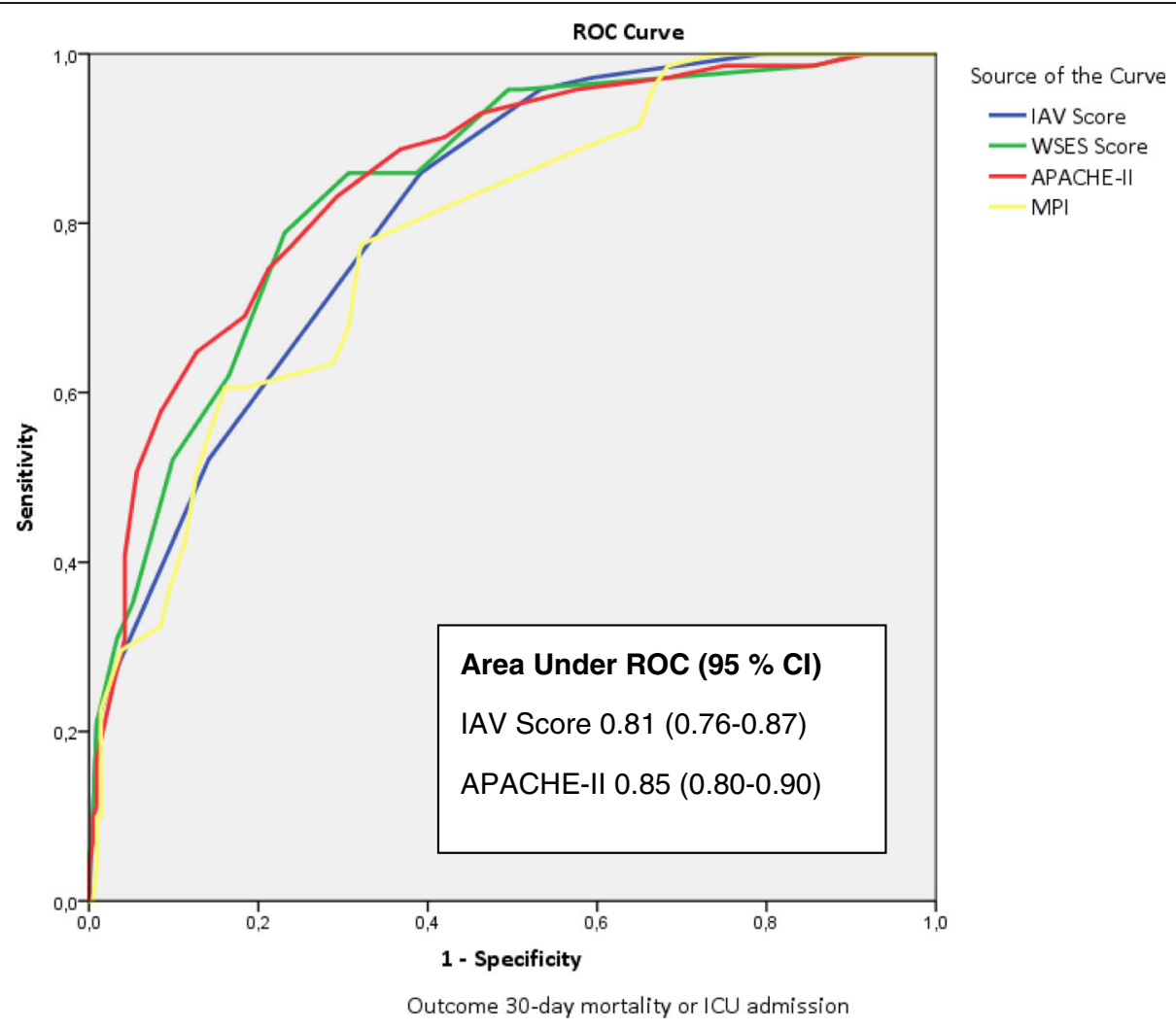

Fig. 2 Receiver operating characteristic (ROC) curve for the intra-abdominal view (IAV) score and comparisons to other scores. Abbreviations: WSES World Society of Emergency Surgery, APACHE Acute Physiology And Chronic Health Evaluation, MPI Mannheim Peritonitis index, Cl confidence interval 
Table 4 The intra-abdominal view (IAV) score evaluation; low, middle, and high scores with various outcomes

\begin{tabular}{|c|c|c|c|c|c|}
\hline & All patients & Low score & Medium score & High score & $P$ value* \\
\hline IAV score & $4(1-4)^{* *}$ & $0-2$ & $3-5$ & $6-7$ & \\
\hline Number of patients, $n$ (\%) & $283(100)$ & $102(36)$ & $158(56)$ & $23(8)$ & \\
\hline Length of stay, days** & $6(3-10)$ & $3(2-5)$ & $8(6-13)$ & $10(6-18)$ & $<0.001$ \\
\hline Clavien-Dindo $\geq 3, n(\%)$ & $87(31)$ & $14(14)$ & $62(39)$ & $11(48)$ & $<0.001$ \\
\hline ICU admission, $n$ (\%) & $57(20)$ & $2(2)$ & $39(25)$ & $16(70)$ & $<0.001$ \\
\hline Mortality, 30 days, $n(\%)$ & $29(10)$ & $1(1)$ & $22(14)$ & $6(26)$ & $<0.001$ \\
\hline SCIAS or 30-day mortality, $n$ (\%) & $71(25)$ & $3(3)$ & $50(32)$ & $17(74)$ & $<0.001$ \\
\hline
\end{tabular}

Abbreviation: ICU intensive care unit

*P values are calculated using linear-by-linear association for dichotomous variables and Kruskal-Wallis test for continuous variable

${ }^{* *}$ Continuous variables are presented as median (interquartile range)

fecal or bile, compared to clear or purulent, there was a more than twofold increase for the primary outcome. This factor is taken into account in the Mannheim Peritonitis Index [2] but not in other cIAI-specific or sepsis scoring systems $[11,13]$.

Fibrin coverage was not independently associated with the primary outcome. Fibrin correlated with redness but the redness was more significant in multivariate analysis and therefore fibrin did not stand out as an independent risk factor. Venous blood flow from the visceral peritoneum goes first through the portal vein into the liver, whereas parietal venous flow goes directly to systemic circulation. Also, the visceral and parietal peritoneum have distinct innervations and the surface area of the visceral peritoneum is much larger than that of the parietal peritoneum [24]. However, localization of the cIAI only in the visceral peritoneum did not correlate with outcome.

The values of Nagelkerke $R^{2} 0.36$ and AUROC 0.81 for the IAV score clearly show that IAV only accounts for a limited, although substantial, part of prognostic factors for SCIAS or 30-day mortality. However, it is quite surprising that the AUROC for the IAV score is of the same magnitude as in the more comprehensive scoring systems, since organ dysfunctions and comorbidities are not included in the model [11]. These results emphasize the role of the surgeons' perception of cIAI disease

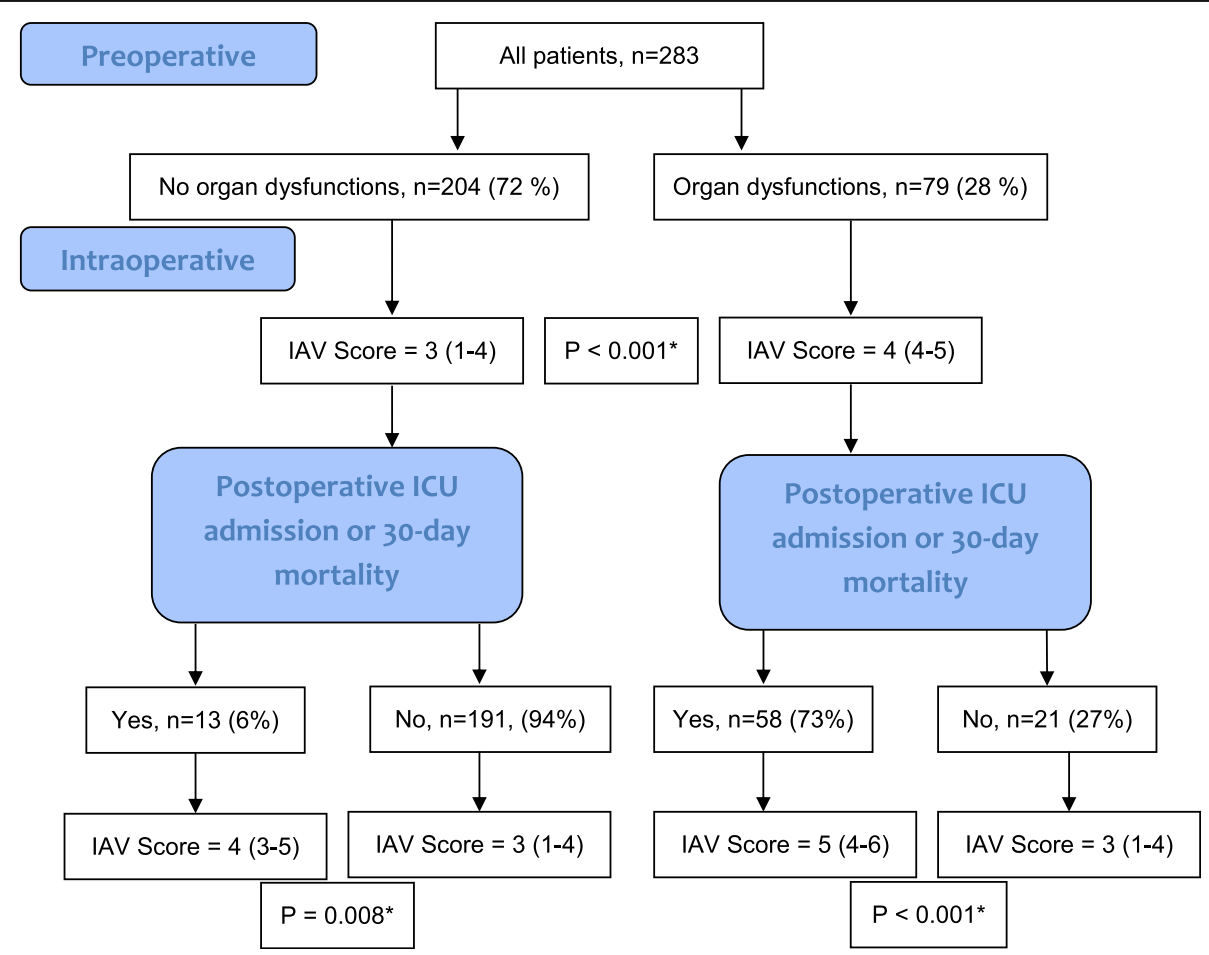

Fig. 3 The intra-abdominal view (IAV) score correlations with pre- and postoperative organ dysfunctions. Legend: IAV scores are presented as median (interquartile range), *Mann-Whitney $U$ test 
severity when looking into the abdomen while operating. A subgroup analysis of patients with a non-appendiceal source of CIAI showed very similar results and was not considered to provide any additional value.

The IAV score was tested by dividing patients into three score groups (Table 4), and it was found to correlate significantly with a variety of different outcomes. In Fig. 3, it is shown that patients who improve quickly, without ICU treatment, from preoperative organ dysfunctions have a lower IAV score. In addition, patients without preoperative organ dysfunctions who eventually develop SCIAS or die have a higher score than patients who recover without organ dysfunctions. It is not surprising that the worse the macroscopic view of the peritonitis is, the worse the outcome. Nevertheless, this is the first study that shows and quantifies it.

Interestingly, in the univariate analyses, when the extent of fibrin and the redness by area was zero, the risk for the primary outcome was high, resulting in a U-shaped curve within the variable. The reason for this finding can only be speculated and it warrants further research, but the phenomenon might be associated with an impaired immune response to cIAI.

It is well known that organ dysfunctions are the most important risk factors for poor outcome $[8,11,13]$. Another major factor not included in this study's model is the comorbidities. These factors were not included in this study since the focus of this study was an in-depth analysis of the IAV. In future studies, including components of the IAV to a more comprehensive scoring system could provide a better scoring system than the current ones.

This study has some limitations. This was a prospective single-center study with a limited number of patients. Less than half of the recruitable patients during the study period were included in the study. The evaluation of the amount of fibrin deposits as well as redness of the peritoneum was based on subjective evaluation leading most likely into some interobserver variability. Also, the IAV score has not been externally validated.

\section{Conclusions}

Classification of peritonitis should not be based only on the type of the exudate or presence of diffuse peritonitis. An IAV score, which includes fecal or bile as exudate, diffuse peritonitis, substantial redness in four or more of the six areas of the abdomen, and non-appendiceal source of cIAI, may provide a simple method to classify patients with cIAI. The IAV score predicts various outcomes well and correlates with preoperative organ dysfunctions and the development of postoperative organ dysfunctions. The concept of including more variables of the IAV to cIAI scoring systems might provide additional value in assessing individual patient disease severity and outcome. However, the IAV score needs external validation before further implementations.

\section{Additional files}

Additional file 1: Paper sheet for the collection of the intra-abdominal findings (DOCX $468 \mathrm{~kb}$ )

Additional file 2: Bootstrapping. Legend: Distribution of AUC value in the bootstrap sample (auc.boot) and the distribution of the AUC value deriving from the model fitted to the bootstrap samples and evaluated on the original sample (auc.orig). The blue line represents apparent AUC and the red line AUC adjusted for optimism. (DOCX 65 kb)

\section{Abbreviations}

APACHE-II: Acute Physiology And Chronic Health Evaluation II; AUROC: Area under the receiver operating characteristic curve; Cl: Confidence interval; CIAI: Complicated intra-abdominal infection; IAV: Intra-abdominal view; ICU: Intensive care unit; IQR: Interquartile range; OR: Odds ratio;

ROC: Receiver operating characteristic; SCIAS: Severe complicated intraabdominal sepsis; SOFA: Sequential Organ Failure Assessment; WSES: World Society of Emergency Surgery

\section{Acknowledgements}

Not applicable.

\section{Funding}

This study was financially supported by Helsinki University Central Hospital Research Funds (Government Research Funds), The Finnish Medical Foundation, Mary and Georg Ehrnrooth's Foundation, and Martti I. Turunen Foundation. The role of the funding body was merely to provide time for researchers to conduct the study.

\section{Availability of data and materials}

The data that supports the findings of this study are available on request from the corresponding author (MT). The data are not publicly available due to institutional data restrictions because the data contains information that could compromise research participant privacy.

\section{Authors' contributions}

MT and MB collected the data. MT had full access to the data in the study and takes responsibility for the collection and integrity of the data and the accuracy of the data analysis. MT drafted the manuscript. All authors (MT, VS, $A L, M B, P M)$ have been involved in the study conception and design, analysis and interpretation of the data, and critical revision of the manuscript. All authors have read and approved the final manuscript and agree to be accountable for all aspects of the work.

\section{Ethics approval and consent to participate}

HUS Helsinki University Hospital operative ethics committee (Dnro 6/13/03/ 02/2016) and review board approved the study design. A written informed consent was obtained from all the patients included in the study.

\section{Consent for publication}

Not applicable.

\section{Competing interests}

The authors declare that they have no competing interests.

\section{Publisher's Note}

Springer Nature remains neutral with regard to jurisdictional claims in published maps and institutional affiliations.

\section{Author details}

${ }^{1}$ Abdominal Center, Department of Abdominal Surgery, Helsinki University Hospital and University of Helsinki, Haartmaninkatu 4, 00290 Helsinki, Finland. 
${ }^{2}$ Abdominal Center, Department of Transplantation and Liver Surgery, Helsinki University Hospital and University of Helsinki, Helsinki, Finland. ${ }^{3}$ Department of Anesthesiology, Intensive Care and Pain Medicine, Division of Intensive Care Medicine, Helsinki University Hospital and University of Helsinki, Helsinki, Finland.

Received: 14 January 2019 Accepted: 5 March 2019

Published online: 29 March 2019

\section{References}

1. Sartelli M, Catena F, Ansaloni L, Coccolini F, Corbella D, Moore EE, et al. Complicated intra-abdominal infections worldwide: the definitive data of the CIAOW study. World J Emerg Surg. 2014;9:37.

2. Linder MM, Wacha H, Feldmann U, Wesch G, Streifensand RA, Gundlach E. The Mannheim peritonitis index. An instrument for the intraoperative prognosis of peritonitis. Chirurg. 1987;58:84-92 3 .

3. Sartelli M, Catena F, Abu-Zidan FM, Ansaloni L, Biffl WL, Boermeester MA, et al. Management of intra-abdominal infections: recommendations by the WSES 2016 consensus conference. World J Emerg Surg. 2017;12:1-32.

4. Hinchey EJ, Schaal PG, Richards GK. Treatment of perforated diverticular disease of the colon. Adv Surg. 1978;12:85-109.

5. Angenete E, Thornell A, Burcharth J, Pommergaard H-C, Skullman S, Bisgaard $T$, et al. Laparoscopic lavage is feasible and safe for the treatment of perforated diverticulitis with purulent peritonitis: the first results from the randomized controlled trial DILALA. Ann Surg. 2016;263:117-22.

6. Sallinen V, Mentula P, Leppaniemi A. Laparoscopic lavage for Hinchey III diverticulitis-but to whom? Ann Surg. 2015;265:e62.

7. Kirkpatrick AW, Coccolini F, Ansaloni L, Roberts DJ, Tolonen M, McKee JL, et al. Closed Or Open after Source Control Laparotomy for Severe Complicated Intra-Abdominal Sepsis (the COOL trial): study protocol for a randomized controlled trial. World J Emerg Surg. 2018;13:26.

8. Tolonen M, Sallinen V, Mentula P, Leppaniemi A. Preoperative prognostic factors for severe diffuse secondary peritonitis: a retrospective study. Langenbeck's Arch Surg. 2016;401:1-7.

9. Pieracci FM, Barie PS. Management of severe sepsis of abdominal origin Scand J Surg. 2007;96:184-96.

10. Singer M, Deutschman CS, Seymour CW, Shankar-Hari M, Annane D, Bauer $M$, et al. The third international consensus definitions for sepsis and septic shock (Sepsis-3). JAMA. 2016;315(8):801-10.

11. Tolonen M, Coccolini F, Ansaloni L, Sartelli M, Roberts DJ, McKee JL, et al. Getting the invite list right: a discussion of sepsis severity scoring systems in severe complicated intra-abdominal sepsis and randomized trial inclusion criteria. World J Emerg Surg. 2018;3:1-11.

12. Charlson ME, Pompei P, Ales KL, Mackenzie CR. A new method of classifying prognostic comorbidity in longitudinal studies: development and validation. J Chronic Dis. 1987;40:373-83.

13. Sartelli M, Abu-Zidan FM, Catena F, Griffiths EA, Di Saverio S, Coimbra R, et al. Global validation of the WSES Sepsis Severity Score for patients with complicated intra-abdominal infections: a prospective multicentre study (WISS study). World J Emerg Surg. 2015;10:1-8.

14. Knaus WA, Draper EA, Wagner DP, Zimmerman JE. APACHE II: a severity of disease classification system. Crit Care Med. 1985;13:818-29.

15. Dindo D, Demartines N, Clavien P-A. Classification of surgical complications: a new proposal with evaluation in a cohort of 6336 patients and results of a survey. Ann Surg. 2004;240:205-13.

16. The SOFA. (Sepsis-related Organ Failure Assessment) score to describe organ dysfunction/failure. On behalf of the Working Group on SepsisRelated Problems of the European Society of Intensive Care Medicine. Intensive Care Med. 1996;22(199):707-10.

17. Mentula PJ, Leppäniemi AK. Applicability of the Clavien-Dindo classification to emergency surgical procedures: a retrospective cohort study on 444 consecutive patients. Patient Saf Surg. 2014;8:31.

18. Steyerberg EW, Harrell FE, Borsboom GJ, Eijkemans MJ, Vergouwe Y, Habbema JD. Internal validation of predictive models: efficiency of some procedures for logistic regression analysis. J Clin Epidemiol. 2001;54(8):774-81.
19. Robin X, Turck N, Hainard A, Tiberti N, Lisacek F, Sanchez JC, et al. pROC: an open-source package for R and S+ to analyze and compare ROC curves. BMC Bioinformatics. 2011;12:77.

20. Alberti G. 'auc.adjust': R function for optimism-adjusted AUC (internal validation); 2016. https://doi.org/10.13140/RG.2.1.1485.0324.

21. Capobianco A, Cottone L, Monno A, Manfredi AA, Rovere-Querini P. The peritoneum: healing, immunity, and diseases. J Pathol. 2017;243:137-47.

22. Sammour T, Kahokehr A, Soop M, Hill AG. Peritoneal damage: the inflammatory response and clinical implications of the neuro-immunohumoral axis. World J Surg. 2010;34:704-20.

23. Broche F, Tellado JM. Defense mechanisms of the peritoneal cavity. Curr Opin Crit Care. 2001;7:105-16.

24. Ross JT, Matthay MA, Harris HW. Secondary peritonitis: principles of diagnosis and intervention. BMJ. 2018;18:k1407-14.

\section{Ready to submit your research? Choose BMC and benefit from:}

- fast, convenient online submission

- thorough peer review by experienced researchers in your field

- rapid publication on acceptance

- support for research data, including large and complex data types

- gold Open Access which fosters wider collaboration and increased citations

- maximum visibility for your research: over $100 \mathrm{M}$ website views per year

At BMC, research is always in progress.

Learn more biomedcentral.com/submissions 